\title{
Nordhaus-Gaddum-type theorems for decompositions into many parts
}

\author{
Zoltan Füredi*1,2 $\quad$ Alexandr V. Kostochka ${ }^{\dagger \dagger 1,3}$ \\ Michael Stiebitz ${ }^{4}$ Riste Škrekovski ${ }^{\S 5,6} \quad$ Douglas B. West ${ }^{\dagger 1}$ \\ ${ }^{1}$ University of Illinois, Urbana, IL 61801, U.S.A. \\ ${ }^{2}$ Rényi Institute of Mathematics, P.O.Box 127, Budapest 1364, Hungary \\ ${ }^{3}$ Institute of Mathematics, 630090 Novosibirsk, Russia \\ 4 Technische Universität Ilmenau, D-98693 Ilmenau, Germany \\ ${ }^{5}$ Charles University, Malostranské nám. 2/25, 118 00, Prague, Czech Republic \\ ${ }^{6}$ University of Ljubljana, Jadranska 19, 1111 Ljubljana, Slovenia
}

\begin{abstract}
A $k$-decomposition $\left(G_{1}, \ldots, G_{k}\right)$ of a graph $G$ is a partition of its edge set to form $k$ spanning subgraphs $G_{1}, \ldots, G_{k}$. The classical theorem of Nordhaus and Gaddum bounds $\chi\left(G_{1}\right)+\chi\left(G_{2}\right)$ and $\chi\left(G_{1}\right) \chi\left(G_{2}\right)$ over all 2-decompositions of $K_{n}$. For a graph parameter $p$, let $p(k ; G)$ denote the maximum of $\sum_{i=1}^{k} p\left(G_{i}\right)$ over all $k$-decompositions of the graph $G$.

The clique number $\omega$, chromatic number $\chi$, list chromatic number $\chi_{\ell}$, and Szekeres-Wilf number $\sigma$ satisfy $\omega\left(2 ; K_{n}\right)=\chi\left(2 ; K_{n}\right)=\chi_{\ell}\left(2 ; K_{n}\right)=$ $\sigma\left(2 ; K_{n}\right)=n+1$. We obtain lower and upper bounds for $\omega\left(k ; K_{n}\right)$, $\chi\left(k ; K_{n}\right), \chi_{\ell}\left(k ; K_{n}\right)$, and $\sigma\left(k ; K_{n}\right)$. The last three behave differently for large $k$. We also obtain lower and upper bounds for the maximum of $\chi(k ; G)$ over all graphs embedded on a given surface.
\end{abstract}

\section{Introduction}

A $k$-decomposition of a (hyper)graph $G$ is a decomposition of $G$ into $k$ spanning sub(hyper)graphs $G_{1}, \ldots, G_{k}$. That is, each $G_{i}$ has the same vertices as $G$, and

\footnotetext{
${ }^{*}$ Research supported in part by the Hungarian National Science Foundation under grant OTKA T 032452, and by the NSF grant DMS 0140692.

${ }^{\dagger}$ Research supported in part by the NSF grant DMS-0099608.

${ }^{\ddagger}$ Research supported in part by the Dutch-Russian grant NWO-047-008-006.

$\S$ Research supported in part by the Ministry of Science and Technology of Slovenia (Z13129), by the Czech Ministry of Education (LN00A056) and by DLR (SVN 99/003).
} 
every edge of $G$ belongs to exactly one of $G_{1}, \ldots, G_{k}$. Such decompositions can be interpreted as unrestricted $k$-edge-colorings of $G$.

For a parameter $p$, a positive integer $k$, and a (hyper)graph $G$, let

$$
p(k ; G)=\max \left\{\sum_{i=1}^{k} p\left(G_{i}\right):\left(G_{1}, \ldots, G_{k}\right) \text { is a } k \text {-decomposition of } G\right\} .
$$

Also, a $p$-optimal $k$-decomposition of $G$ is a $k$-decomposition $\left(G_{1}, \ldots, G_{k}\right)$ such that $p(k ; G)=\sum_{i=1}^{k} p\left(G_{i}\right)$.

The parameters that interest us are the clique number $\omega$, the chromatic number $\chi$, the list-chromatic number $\chi_{\ell}$, and the Szekeres-Wilf number $\sigma$, where $\sigma$ is defined by $\sigma(G)=1+\max _{H \subseteq G} \delta(H)$. Every graph $G$ satisfies $\omega(G) \leq \chi(G) \leq$ $\chi_{\ell}(G) \leq \sigma(G)$. Therefore, for every $k$ and $G$,

$$
\omega(k ; G) \leq \chi(k ; G) \leq \chi_{\ell}(k ; G) \leq \sigma(k ; G) .
$$

Clearly, $\left(G_{1}, G_{2}\right)$ is a 2-decomposition of the complete graph $K_{n}$ if and only if $G_{1}$ is the complement of $G_{2}$ and has $n$ vertices. Thus the Nordhaus-Gaddum Theorem can be stated as follows.

Theorem 1 (Nordhaus-Gaddum [15]) Let $n$ be a positive integer. If $\left(G_{1}, G_{2}\right)$ is a 2-decomposition of $K_{n}$, then the following statements hold:

(a) $\lceil 2 \sqrt{n}\rceil \leq \chi\left(G_{1}\right)+\chi\left(G_{2}\right) \leq n+1$

(b) $n \leq \chi\left(G_{1}\right) \cdot \chi\left(G_{2}\right) \leq\left\lfloor\left(\frac{n+1}{2}\right)^{2}\right\rfloor$.

The proof of Theorem 1 implies that $\omega\left(2 ; K_{n}\right)=\chi\left(2 ; K_{n}\right)=\chi_{\ell}\left(2 ; K_{n}\right)=$ $\sigma\left(2 ; K_{n}\right)=n+1$, achieving equalities in (1) (see also Section 5). Finck [9] described the $\chi$-optimal 2-decompositions of $K_{n}$.

Plesník [16] studied $\chi\left(k ; K_{n}\right)$ for $k>2$ and proved that $\chi\left(k ; K_{n}\right) \leq n+2^{\left(\begin{array}{c}k+1 \\ 2\end{array}\right)}$ for every $n$. He also proved (see Lemma 3 in [16]) that

$$
n+\left(\begin{array}{l}
k \\
2
\end{array}\right) \leq \omega\left(k ; K_{n}\right) \leq \chi\left(k ; K_{n}\right) \quad \text { if } \quad n \geq\left(\begin{array}{l}
k \\
2
\end{array}\right)
$$

and conjectured (see also Bosák [3]) that $\chi\left(k ; K_{n}\right)=n+\left(\begin{array}{c}k \\ 2\end{array}\right)$. Our first result is

Theorem 2 If $k$ and $n$ are positive integers, then $\omega\left(k ; K_{n}\right) \leq n+\left(\begin{array}{l}k \\ 2\end{array}\right)$. If $n \geq\left(\begin{array}{l}k \\ 2\end{array}\right)$, then $\omega\left(k ; K_{n}\right)=n+\left(\begin{array}{l}k \\ 2\end{array}\right)$.

Watkinson [23] improved Plesník's upper bound to $\chi\left(k ; K_{n}\right) \leq n+\frac{k !}{2}$. It follows that $\omega\left(3 ; K_{n}\right)=\chi\left(3 ; K_{n}\right)=n+3$ for every $n \geq 3$. Our second result improves Watkinson's bound for large $k$.

Theorem 3 If $k$ and $n$ are positive integers, then $\chi\left(k ; K_{n}\right) \leq n+7^{k}$. 
The list chromatic number behaves somewhat differently for large $k$.

Theorem 4 There exists a positive constant $c$ such that, if $k=\left(\begin{array}{c}\ell+1 \\ 2\end{array}\right)$ and $n=\ell m$ where $\ell$ and $m$ are integers greater than 1 , then

$$
\chi_{\ell}\left(k ; K_{n}\right) \geq n+c k \ln \left(\frac{n}{\sqrt{k}}\right) .
$$

On the other hand, for all positive integers $k$ and $n$,

$$
\chi_{\ell}\left(k ; K_{n}\right) \leq n+3 k ! \sqrt{1+8 n \ln n} .
$$

Thus, the leading behavior of $\chi_{\ell}\left(k, K_{n}\right)$ for fixed $k$ as $n \rightarrow \infty$ is still $n$, but there is an additive term growing with $n$. The situation with $\sigma\left(k ; K_{n}\right)$ is different.

Theorem 5 If $k=p^{2}+p+1$ for some prime power $p$, and $n \equiv 0 \bmod k$, then

$$
\sigma\left(k ; K_{n}\right) \geq(\sqrt{k}-1) n+k,
$$

On the other hand, for all positive integers $k$ and $n$,

$$
\sigma\left(k ; K_{n}\right) \leq \sqrt{k} n+k .
$$

Furthermore, we determine $\sigma\left(k ; K_{n}\right)$ exactly for $k \leq 4$.

We consider also decompositions of the complete $r$-uniform hypergraph $K_{n}^{r}$. Since $\chi\left(K_{n}^{r}\right)=\left\lceil\frac{n}{r-1}\right\rceil$, we have $\chi\left(k ; K_{n}^{r}\right) \geq\left\lceil\frac{n}{r-1}\right\rceil+k-1$. Although it is easy to improve the bound for large $n$, we will prove that up to a summand $c_{k, r}$ independent of $n$, this is the correct value of $\chi\left(k ; K_{n}^{r}\right)$.

Theorem 6 If $k$ and $r$ are positive integers with $r \geq 2$, then there exists an integer $c_{k, r}$ such that, for every positive integer $n$,

$$
\chi\left(k ; K_{n}^{r}\right) \leq \frac{n}{r-1}+c_{k, r}
$$

Finally, we consider decompositions of graphs embedded on a given surface $\Sigma$. For a graph parameter $p$ and positive integer $k$, let $p(k ; \Sigma)$ denote the maximum of $p(k ; G)$ over all graphs $G$ embeddable on $\Sigma$. Let $g$ be the Euler genus of $\Sigma$. We show that for fixed $k$ and large $g$, the values of $\omega(k ; \Sigma), \chi(k ; \Sigma), \chi_{\ell}(k ; \Sigma)$, and $\sigma(k ; \Sigma)$ are asymptotically equal, unlike $\omega\left(k ; K_{n}\right)$ and $\sigma\left(k ; K_{n}\right)$.

Along with the maximum sum, we can also study the maximum product of the values of a parameter $p$ over a decomposition. Let

$$
\widetilde{p}(k ; G)=\max \left\{\prod_{i=1}^{k} p\left(G_{i}\right):\left(G_{1}, \ldots, G_{k}\right) \text { is a } k \text {-decomposition of } G\right\} .
$$


Using the Cauchy-Schwarz Inequality, our bounds on $p\left(k ; K_{n}\right)$ yield corresponding bounds on $\widetilde{p}\left(k ; K_{n}\right)$. For fixed $k$,

$$
\begin{gathered}
\widetilde{\omega}\left(k ; K_{n}\right) \leq \widetilde{\chi}\left(k ; K_{n}\right) \leq \widetilde{\chi_{\ell}}\left(k ; K_{n}\right) \leq(1+o(1))(n / k)^{k} \\
\widetilde{\chi}\left(k ; K_{n}^{r}\right) \leq(1+o(1))(n / k(r-1))^{k}, \quad \text { and } \\
\widetilde{\sigma}\left(k ; K_{n}\right) \leq(1+o(1))(n / \sqrt{k})^{k}
\end{gathered}
$$

Decomposing $K_{n}$ and $K_{n}^{r}$ into almost disjoint complete subgraphs of about the same size (pairwise sharing at most $r-1$ vertices) shows that except for the bound on $\tilde{\sigma}$, these upper bounds are asymptotically tight for fixed $k$. The construction in Theorem 5 yields that $\widetilde{\sigma}\left(k ; K_{n}\right) \geq e^{-\sqrt{k}}(n / \sqrt{k})^{k}$. Thus, for $\widetilde{\sigma}\left(k ; K_{n}\right)$ we at least know the order of magnitude.

In the next section, we summarize notation and terminology. In subsequent sections we treat consecutively the clique number, the chromatic number, the list chromatic number, and the Szekeres-Wilf number of graphs. The last two sections are devoted to the chromatic number of $r$-uniform hypergraphs and to decompositions of graphs embedded on surfaces.

\section{Preliminaries}

Concepts and notation not defined in this paper are as in standard textbooks. Though the main objects of our study are graphs, we also consider the central concepts for hypergraphs.

A hypergraph $G$ is a pair consisting of a finite set $V(G)$ of vertices and a set $E(G)$ of subsets of $V(G)$, called edges, each having size at least two. A hypergraph $G$ is $r$-uniform if $|e|=r$ for every $e \in E(G)$. A graph is a 2-uniform hypergraph.

Let $G$ be a hypergraph. The degree $d_{G}(x)$ of a vertex $x \in V(G)$ is the number of edges in $G$ that contain $x$. If $d_{G}(x)=s$ for every vertex $x \in V(G)$, then $G$ is $s$-regular. Let $\delta(G)$ denote the minimum degree of $G$.

If $H$ and $G$ are hypergraphs with $V(H) \subseteq V(G)$ and $E(H) \subseteq E(G)$, then $H$ is a subhypergraph of $G$. In this case we write $H \subseteq G$.

Let $G$ be a hypergraph. For $X \subseteq V(G)$, the subhypergraph of $G$ induced by $X$, written $G[X]$, is defined by $V(G[X])=X$ and $E(G[X])=\{e \in E(G): e \subseteq X\}$. Let $G-X$ denote $G[V(G)-X]$.

For an $r$-uniform hypergraph $G$, a set $X \subseteq V(G)$ is a clique or an independent set if $E(G[X])$ consists of all $r$-subsets of $X$ or is empty, respectively. For an $r$-uniform hypergraph $G$, the clique number $\omega(G)$ is the maximum size of a clique in $G$. We write $K_{n}^{r}$ for an $r$-uniform complete hypergraph on $n$ vertices (the edges are all $r$-subsets of the vertices). Thus $K_{n}^{2}$ denotes simply a complete graph $K_{n}$.

For a (hyper)graph $G$, a list assignment $L$ is a function that assigns to each vertex $x$ of $G$ a set $L(x)$ of colors (positive integers). An $L$-coloring of $G$ is a 
function $c$ that assigns a color to each vertex of $G$ such that $c(x) \in L(x)$ for all $x \in V(G)$ and $|\{c(x): x \in e\}| \geq 2$ for each $e \in E(G)$. If $G$ admits an $L$-coloring, then $G$ is $L$-colorable. When $L(x)=[k]$ for all $x \in V(G)$ (where $[k]$ denotes the set $\{1, \ldots, k\})$, the corresponding terms become $k$-coloring and $k$-colorable, respectively. $G$ is $k$-list-colorable if $G$ is $L$-colorable for every list assignment $L$ satisfying $|L(x)|=k$ for all $x \in V(G)$.

The chromatic number of $G$, denoted by $\chi(G)$, is the least $k$ such that $G$ is $k$-colorable. The list-chromatic number of $G$, denoted by $\chi_{\ell}(G)$, is the least $k$ such that $G$ is $k$-list-colorable.

The Szekeres-Wilf number $\sigma(G)$ of a graph $G$, introduced by Szekeres and Wilf [20] in 1968, is equal to the coloring number introduced and studied by Erdös and Hajnal [7] in 1966. It is the smallest integer $d$ such that in some linear ordering of $V(G)$ every vertex of $G$ has at most $d-1$ neighbors following it. It has been observed repeatedly that $\sigma(G)$ can be easily computed by iteratively letting $x_{i}$ be a vertex of minimum degree in the subgraph $G_{i}$ obtained by deleting $\left\{x_{j}: j<i\right\}$. It follows that $\sigma(G)=1+\max _{i}\left\{\delta\left(G_{i}\right)\right\}$.

Let $p$ be a (hyper)graph parameter. A graph $G$ is $p$-critical if $p(H)<p(G)$ for every proper subgraph $H$ of $G$. For $p \in\left\{\omega, \chi, \chi_{\ell}, \sigma\right\}$, every graph $G$ contains a $p$-critical subgraph $H$ satisfying $p(H)=p(G)$.

\section{Clique number and chromatic number}

In discussing $\omega\left(k ; K_{n}\right)$, we noted that a lower bound of $n+\left(\begin{array}{l}k \\ 2\end{array}\right)$ is proved in [16] for the case $n \geq\left(\begin{array}{l}k \\ 2\end{array}\right)$. We include a simple construction for this before proving our upper bound.

Construction: If $n \geq\left(\begin{array}{l}k \\ 2\end{array}\right)$, then $\omega\left(k ; K_{n}\right) \geq n+\left(\begin{array}{l}k \\ 2\end{array}\right)$. The construction for $n=\left(\begin{array}{c}k \\ 2\end{array}\right)$ can be extended for each additional vertex by adding all the edges incident to the new vertex to one graph in the decomposition.

For $n=\left(\begin{array}{l}k \\ 2\end{array}\right)$, we provide $k$ cliques of order $k-1$ that are pairwise edge-disjoint and together use each vertex twice. The edges not covered can be added to one of these subgraphs to complete the decomposition of $K_{n}$. The sum of the orders of the cliques is $k(k-1)$, which equals $n+\left(\begin{array}{l}k \\ 2\end{array}\right)$.

Let $V\left(K_{n}\right)=\{(i, j): 1 \leq i<j \leq k\}$. For $1 \leq r \leq k$, let $Q_{r}$ be the set of vertices whose names have $r$ in one coordinate; note that $\left|Q_{r}\right|=k-1$. The only vertex shared by $Q_{i}$ and $Q_{j}$ with $i<j$ is $(i, j)$, so the cliques are edge-disjoint.

Theorem 2 If $k$ and $n$ are positive integers, then $\omega\left(k ; K_{n}\right) \leq n+\left(\begin{array}{l}k \\ 2\end{array}\right)$. If $n \geq\left(\begin{array}{l}k \\ 2\end{array}\right)$, then $\omega\left(k ; K_{n}\right)=n+\left(\begin{array}{l}k \\ 2\end{array}\right)$. 
Proof: By the construction above, it suffices to show that $\omega\left(k ; K_{n}\right) \leq n+\left(\begin{array}{c}k \\ 2\end{array}\right)$. We prove this by induction on $n+k$. For $n=1$, we have $\omega\left(k ; K_{1}\right)=k \leq 1+\left(\begin{array}{l}k \\ 2\end{array}\right)$. For $k=1$, we have $\omega\left(1 ; K_{n}\right)=n=n+\left(\begin{array}{l}1 \\ 2\end{array}\right)$.

For $\min \{k, n\} \geq 2$, let $\left(G_{1}, \ldots, G_{k}\right)$ be an $\omega$-optimal $k$-decomposition of $K_{n}$. For $1 \leq i \leq k$, let $X_{i}$ denote a maximum clique of $G_{i}$.

If $\omega\left(G_{1}\right)=\left|X_{1}\right| \leq k-1$, then since $\left(G_{1} \cup G_{2}, G_{3}, \ldots, G_{k}\right)$ is a $(k-1)$ decomposition of $K_{n}$, it follows from the induction hypothesis that

$$
\begin{aligned}
\omega\left(k ; K_{n}\right) & =\sum_{i=1}^{k} \omega\left(G_{i}\right) \leq(k-1)+\omega\left(k-1 ; K_{n}\right) \\
& \leq(k-1)+n+\left(\begin{array}{c}
k-1 \\
2
\end{array}\right)=n+\left(\begin{array}{c}
k \\
2
\end{array}\right)
\end{aligned}
$$

Otherwise, since $\left|X_{1} \cap X_{j}\right| \leq 1$ for $2 \leq j \leq k$, we can choose a vertex $x \in$ $X_{1}-\bigcup_{j=2}^{k} X_{j}$. Now $\left(G_{1}-x, \ldots, G_{k}-x\right)$ is a $k$-decomposition of $K_{n-1}$. By the induction hypothesis, it follows that

$$
\begin{aligned}
\omega\left(k ; K_{n}\right) & =\sum_{i=1}^{k} \omega\left(G_{i}\right) \leq 1+\sum_{i=1}^{k} \omega\left(G_{i}-x\right) \\
& \leq 1+\omega\left(k ; K_{n-1}\right) \leq 1+(n-1)+\left(\begin{array}{l}
k \\
2
\end{array}\right)=n+\left(\begin{array}{l}
k \\
2
\end{array}\right) .
\end{aligned}
$$

To obtain our upper bound on $\chi\left(k ; K_{n}\right)$, we need an upper bound on chromatic number in terms of Ramsey numbers. In our language, Ramsey's Theorem states that every $k$-decomposition of a sufficiently large $r$-uniform complete hypergraph has an $r$-uniform complete hypergraph of specified size in some factor.

Theorem 7 (Ramsey [19]) For positive integers $k, r$, and $n_{1}, \ldots, n_{k}$ satisfying $k, r \geq 2$, there is a smallest integer $R$ (written as $R_{k}^{r}\left(n_{1}, \ldots, n_{k}\right)$ ) such that if $\left(G_{1}, \ldots, G_{k}\right)$ is a $k$-decomposition of $K_{n}^{r}$ with $n \geq R$, then for some $i \in[k]$ the subhypergraph $G_{i}$ contains an r-uniform complete hypergraph with $n_{i}$ vertices.

Our bound uses the observation that proper colorings of the subgraphs induced by a partition of the vertices, with different colors, combine to form a proper coloring of the full graph. We call this subadditivity of $\chi$.

Proposition 8 For positive integers $k, \ell$, and $r$ with $r \geq 2$, let $R$ be the Ramsey number $R_{2}^{r}(\ell, k)$. If $G$ is an $r$-uniform n-vertex hypergraph containing no $r$ uniform complete hypergraph on $\ell$ vertices, then

$$
\chi(G) \leq \frac{n-R}{k}+R .
$$


Proof: We use induction on $n$. If $n \leq R$, then

$$
\chi(G) \leq n=\frac{n}{k}+n \frac{k-1}{k} \leq \frac{n}{k}+R \frac{k-1}{k} \leq \frac{n-R}{k}+R .
$$

Now consider $n>R$. By the definition of the Ramsey number, $G$ has an independent set $X$ with $|X| \geq k$. By the induction hypothesis and subadditivity of $\chi$,

$$
\chi(G) \leq \chi(G-X)+1 \leq \frac{n-|X|-R}{k}+R+1 \leq \frac{n-R}{k}+R .
$$

In Theorem 3, our attention is on ordinary graphs, and we use only the case $r=2$ of Proposition 8 .

Theorem 3 If $k$ and $n$ are positive integers, then $\chi\left(k ; K_{n}\right) \leq n+7^{k}$.

Proof: The claim is trivial for $n=1$ or $k=1$. For $k>1$, we use induction on $n$. For the induction step, consider $n \geq 2$. Let $\left(G_{1}, \ldots, G_{k}\right)$ be a $k$-decomposition of $K_{n}$. To show that $\sum_{i=1}^{k} \chi\left(G_{i}\right) \leq n+7^{k}$, we distinguish two cases.

Case 1: In some subgraph of the decomposition, there is a maximum clique whose deletion leaves a clique of size at least $2 k-2$. We may assume that this occurs in $G_{1}$. Thus $G_{1}$ has a maximum clique $X$ such that $\omega\left(G_{1}-X\right) \geq 2 k-2$. Let $Y$ be a maximum clique of $G_{1}-X$, and let $Z=X \cup Y$. The subgraph $G_{1}[Z]$ is the complement of a bipartite graph, and each $G_{i}[Z]$ for $i \geq 2$ is bipartite. Hence all these subgraphs are perfect. Moreover, $\chi\left(G_{1}[Z]\right)=\omega\left(G_{1}[Z]\right)=|X|$, and $\chi\left(G_{i}[Z]\right) \leq 2$ for $2 \leq i \leq k$. Thus

$$
\sum_{i=1}^{k} \chi\left(G_{i}[Z]\right) \leq|X|+2(k-1) \leq|X|+|Y|=|Z| .
$$

Furthermore, $\left(G_{1}-Z, \ldots, G_{k}-Z\right)$ is a $k$-decomposition of $K_{n-|Z|}$. By the induction hypothesis, $\sum_{i=1}^{k} \chi\left(G_{i}-Z\right) \leq n-|Z|+7^{k}$. Using subadditivity,

$$
\sum_{i=1}^{k} \chi\left(G_{i}\right) \leq \sum_{i=1}^{k} \chi\left(G_{i}[Z]\right)+\sum_{i=1}^{k} \chi\left(G_{i}-Z\right) \leq|Z|+n-|Z|+7^{k}=n+7^{k} .
$$

Case 2: For each $i$, if $X$ is a maximum clique of $G_{i}$, then $\omega\left(G_{i}-X\right) \leq 2 k-3$. Define a sequence of subsets of $V\left(K_{n}\right)$ as follows. Let $X_{0}=\emptyset$. For $j \geq 1$, let $X_{j}$ be a maximum clique among the graphs $G_{1}-\bigcup_{i=0}^{j-1} X_{i}, \ldots, G_{k}-\bigcup_{i=0}^{j-1} X_{i}$.

Let $\ell_{j}$ be the index $i$ such that $X_{j}$ is a clique in $G_{i}$. For $i \neq \ell_{j}, X_{j}$ is an independent set in $G_{i}$. For $j \geq 1$, this yields $\sum_{i=1}^{k} \chi\left(G_{i}\left[X_{j}\right]\right) \leq\left|X_{j}\right|+k-1$. Let 
$p$ be the least index such that $\ell_{p} \in\left\{\ell_{1}, \ldots, \ell_{p-1}\right\}$. Let $X=X_{1} \cup \cdots \cup X_{p-1}$, and let $m=|X|$. By subadditivity,

$$
\sum_{i=1}^{k} \chi\left(G_{i}[X]\right) \leq|X|+(p-1)(k-1) \leq m+k(k-1) .
$$

Let $G_{i}^{\prime}=G_{i}-X$ for each $i$. Since the second clique taken from $G_{p}$ has size at most $2 k-3$ and is a largest clique remaining in any subgraph when it is chosen, $\omega\left(G_{i}^{\prime}\right) \leq 2 k-3$ for all $i$. Let $R=R_{2}^{2}(k, 2 k-2)$. By Proposition 8 , $\chi\left(G_{i}^{\prime}\right) \leq \frac{n-m-R}{k}+R$ for each $i$. Therefore,

$$
\sum_{i=1}^{k} \chi\left(G_{i}^{\prime}\right) \leq n-m-R+k R=n-m+(k-1) R .
$$

By subadditivity,

$$
\sum_{i=1}^{k} \chi\left(G_{i}\right) \leq m+k(k-1)+n-m+(k-1) R=n+(k-1)(k+R) .
$$

It remains only to supply an upper bound on $R$. From the well-known bound $R_{2}^{2}(p, q) \leq\left(\begin{array}{c}p+q-2 \\ p-1\end{array}\right)$, we have $R=R_{2}^{2}(k, 2 k-2) \leq\left(\begin{array}{c}3 k-4 \\ k-1\end{array}\right)$. Let $t=k-1$. From Stirling's Formula for factorials, $\left(\begin{array}{c}3 t-1 \\ t\end{array}\right)$ is approximately $(3 \pi t)^{-1 / 2}\left(\frac{27}{4}\right)^{t}$. For all $k$, we have $(k-1)(k+R)<7^{k}$, and the desired bound follows.

The proof of Theorem 3 shows that in fact $n+O\left(\sqrt{k}\left(\frac{27}{4}\right)^{k}\right)$ is an upper bound. As was mentioned in the introduction, $\omega\left(k ; K_{n}\right)=\chi\left(k ; K_{n}\right)$ for $k \leq 3$. We do not know whether $\omega\left(k ; K_{n}\right)=\chi\left(k ; K_{n}\right)$ for any larger $k$.

\section{The list-chromatic number}

The list-chromatic number is not subadditive, but a weaker statement holds.

Lemma 9 If $H$ is a graph with at most $n$ vertices, and $X_{1}, \ldots, X_{k}$ are disjoint sets with union $V(H)$, then

$$
\chi_{\ell}(H) \leq \sum_{i=1}^{k} \chi_{\ell}\left(H\left[X_{i}\right]\right)+k \sqrt{8 n \ln n}
$$


Proof: If any $X_{i}$ is empty, then we can delete it, reduce $k$, and obtain a better upper bound. Hence we may assume that $X_{1}, \ldots, X_{k}$ are nonempty, which requires $k \leq n$. Since $\chi_{\ell}(H) \leq n$, the statement is trivial if $n \leq \sqrt{8 n \ln n}$, so we may also assume that $n>\sqrt{8 n \ln n}$. Let $q=k \sqrt{8 n \ln n}$, so $n>q / k$.

Let $L$ be a list assignment for $H$ such that $|L(x)|=m$ for all $x \in V(H)$, where $m$ is the ceiling of the claimed upper bound. We show that $H$ is $L$ colorable. To prove this, we use a probabilistic argument due to Alon [2]. Let $S=\bigcup_{x \in V(H)} L(x)$. The idea is to restrict each color in $S$ to be used on only one $H\left[X_{i}\right]$ and show that some such restriction yields an $L$-coloring.

For $1 \leq i \leq k$, let $m_{i}=\chi_{\ell}\left(H\left[X_{i}\right]\right)$, and let $p_{i}=\frac{m_{i}+q / k}{m}$. Thus $0 \leq p_{i} \leq 1$ and $p_{1}+\ldots+p_{k}=1$. For each $s \in S$, independently, place $s$ into one of $S_{1}, \ldots, S_{k}$, letting the choice be $S_{i}$ with probability $p_{i}$.

For each graph $H\left[X_{i}\right]$, define a list assignment $L_{i}$ by letting $L_{i}(x)=L(x) \cap S_{i}$ for each $x \in X_{i}$. The list assignment $L_{i}$ is $\operatorname{good}$ if $\left|L_{i}(x)\right| \geq m_{i}$ for all $x \in X_{i}$. If $L_{i}$ is good, then $H\left[X_{i}\right]$ is $L_{i}$-colorable. If each $H\left[X_{i}\right]$ is $L_{i}$-colorable, then $H$ is $L$ colorable. Therefore, it suffices to show that for some outcome of the experiment each $L_{i}$ is good.

Fix $i$ and $x \in X_{i}$, and let $\lambda=\mathbf{E}\left(\left|L_{i}(x)\right|\right)$. Note that $\lambda=m p_{i}=m_{i}+q / k$. By Chernoff's inequality (see Theorem 2.1 in [12]), we obtain

$$
\operatorname{Pr}\left(\left|L_{i}(x)\right|<m_{i}\right)=\operatorname{Pr}\left(\left|L_{i}(x)\right|<\lambda-q / k\right) \leq e^{-(q / k)^{2} / 2 \lambda} .
$$

Since $n>q / k$, we have $\lambda=m_{i}+q / k \leq n+q / k<2 n$. Therefore,

$$
e^{-(q / k)^{2} / 2 \lambda}<e^{-8 n \ln n / 4 n}=\frac{1}{n^{2}} \leq \frac{1}{k n} .
$$

Since $\operatorname{Pr}\left(\left|L_{i}(x)\right|<m_{i}\right)<1 /(k n)$, and we want to avoid $k n$ such events, there is an outcome of the experiment such that each $L_{i}$ is good.

Now we are ready to prove Theorem 4.

Theorem 4 There exists a positive constant c such that, if $k=\left(\begin{array}{c}\ell+1 \\ 2\end{array}\right)$ and $n=\ell m$ with $\ell$ and $m$ being integers greater than 1 , then

$$
\chi_{\ell}\left(k ; K_{n}\right) \geq n+c k \ln (n / \sqrt{k}) .
$$

On the other hand, for all positive integers $k$ and $n$,

$$
\chi_{\ell}\left(k ; K_{n}\right) \leq n+3 k ! \sqrt{1+8 n \ln n} .
$$

Proof: We provide a construction for the lower bound. For $k$ and $n$ of the given form, $K_{n}$ has a $k$-decomposition using $\ell$ graphs isomorphic to $K_{m}$ and $\left(\begin{array}{l}\ell \\ 2\end{array}\right)$ 
graphs isomorphic to the complete bipartite graph $K_{m, m}$. By a result of Alon [2], $\chi_{\ell}\left(K_{m, m}\right) \geq c_{1} \ln m$ for some positive constant $c_{1}$. Also $\chi_{\ell}\left(K_{m}\right)=m$, so

$$
\chi_{\ell}\left(k ; K_{n}\right) \geq \ell m+c_{1}\left(\begin{array}{l}
\ell \\
2
\end{array}\right) \ln m \geq n+c_{1}(k-l) \ln \left(\frac{n}{l}\right) \geq n+0.5 c_{1} k \ln \left(\frac{n}{\sqrt{k}}\right) .
$$

For the upper bound, we first define $a_{k}$ for $k \geq 1$ by

$$
a_{k}= \begin{cases}3-e & \text { if } k=1 \\ k !\left(a_{1}+\sum_{j=0}^{k-2} \frac{1}{j !}\right) & \text { if } k \geq 2 .\end{cases}
$$

For $k \geq 2$, we have $a_{k}=k(k-1)+k a_{k-1}$ and $k ! \leq a_{k} \leq 3 k$ !. Therefore, it suffices to show that $\chi_{\ell}\left(k ; K_{n}\right) \leq n+a_{k} \sqrt{1+8 n \ln n}$, which we prove by induction on $k$. The inequality is trivial for $k=1$.

Suppose that $k \geq 2$. Let $\left(G_{1}, \ldots, G_{k}\right)$ be a $\chi$-optimal $k$-decomposition of $K_{n}$. For each $i$, let $H_{i}$ be a $\chi_{\ell}$-critical subgraph of $G_{i}$ with $\chi_{\ell}\left(H_{i}\right)=\chi_{\ell}\left(G_{i}\right)$; note that $\delta\left(H_{i}\right) \geq \chi_{\ell}\left(H_{i}\right)-1$. If some vertex $x$ belongs to every $H_{i}$, then

$\chi_{\ell}\left(k ; K_{n}\right)=\sum_{i=1}^{k} \chi_{\ell}\left(H_{i}\right) \leq \sum_{i=1}^{k}\left(d_{H_{i}}(x)+1\right) \leq n-1+k \leq n+a_{k} \sqrt{1+8 n \ln n}$.

Otherwise, each vertex avoids some $H_{j}$, so we can choose disjoint $X^{1}, \ldots, X^{k}$ with union $V\left(K_{n}\right)$ such that $X^{j} \cap V\left(H_{j}\right)=\emptyset$ for all $j$. Now let $K^{j}=K_{n}\left[X^{j}\right]$ and $H_{i}^{j}=H_{i}\left[X^{j}\right]$. Since $X^{j}$ avoids $H_{j}$ and $H_{1}, \ldots, H_{k}$ are pairwise edge-disjoint, $\left(H_{1}^{j}, \ldots, H_{j-1}^{j}, H_{j+1}^{j}, \ldots, H_{k}^{j}\right)$ is a $(k-1)$-decomposition of $K^{j}$. With $n_{j}=\left|X^{j}\right|$, the induction hypothesis yields

$$
\begin{aligned}
\sum_{i} \chi_{\ell}\left(H_{i}^{j}\right) & \leq \chi_{\ell}\left(k-1 ; K_{n_{j}}\right) \leq n_{j}+a_{k-1} \sqrt{1+8 n_{j} \ln n_{j}} \\
& \leq n_{j}+a_{k-1} \sqrt{1+8 n \ln n} .
\end{aligned}
$$

For fixed $i$, on the other hand, Lemma 9 implies that

$$
\chi_{\ell}\left(H_{i}\right) \leq \sum_{j} \chi_{\ell}\left(H_{i}^{j}\right)+(k-1) \sqrt{1+8 n \ln n} .
$$

Consequently,

$$
\begin{aligned}
\chi_{\ell}\left(k ; K_{n}\right) & =\sum_{i=1}^{k} \chi_{\ell}\left(H_{i}\right) \leq \sum_{j=1}^{k} n_{j}+k\left(a_{k-1}+(k-1)\right) \sqrt{1+8 n \ln n} \\
& \leq n+a_{k} \sqrt{1+8 n \ln n} .
\end{aligned}
$$




\section{The Szekeres-Wilf number}

Recall that $\sigma(G)=1+\max _{H \subseteq G} \delta(H)$.

Theorem 5 If $k=p^{2}+p+1$ for some prime power $p$, and $n \equiv 0 \bmod k$, then

$$
\sigma\left(k ; K_{n}\right) \geq(\sqrt{k}-1) n+k,
$$

On the other hand, for all positive integers $k$ and $n$,

$$
\sigma\left(k ; K_{n}\right) \leq \sqrt{k} n+k .
$$

Proof: For the upper bound, let $\left(G_{1}, \ldots, G_{k}\right)$ be a $k$-decomposition of $K_{n}$. Let $d_{i}=\sigma\left(G_{i}\right)$ and $D=\sum_{i=1}^{k} d_{i}$. We show that $D \leq \sqrt{k} n+k$. Each $G_{i}$ has a subgraph $H_{i}$ such that $d_{i}=\delta\left(H_{i}\right)-1$. Thus $\left|E\left(G_{i}\right)\right| \geq\left|E\left(H_{i}\right)\right| \geq\left(\begin{array}{c}d_{i} \\ 2\end{array}\right)$. Since $G_{1}, \ldots, G_{k}$ are edge-disjoint subgraphs of $K_{n}$, we obtain

$$
\frac{n^{2}}{2} \geq\left(\begin{array}{l}
n \\
2
\end{array}\right) \geq \sum_{i=1}^{k}\left(\begin{array}{l}
d_{i} \\
2
\end{array}\right)=\frac{1}{2} \sum_{i=1}^{k}\left(d_{i}^{2}-d_{i}\right) \geq \frac{1}{2}\left(\frac{D^{2}}{k}-D\right) .
$$

Consequently, $D^{2}-k D-k n^{2} \leq 0$, and thus $D \leq \frac{k}{2}+\sqrt{\frac{k^{2}}{4}+k n^{2}} \leq k+\sqrt{k} n$.

We provide a construction for the lower bound. Let $p$ be a prime power, and let $k=p^{2}+p+1$ and $n=m k$ for some integer $m \geq 1$. There is a projective plane with points $[k]$ and lines $\left\{g_{1}, \ldots, g_{k}\right\}$. Partition $V\left(K_{n}\right)$ into sets $X_{1}, \ldots, X_{k}$ of size $m$. Each line $g_{i}$ is a subset of $[k]$; let $H_{i}$ be the complete $(p+1)$ partite graph whose color classes are the elements of $\left\{X_{1}, \ldots, X_{k}\right\}$ indexed by $g_{i}$. The graphs $H_{1}, \ldots, H_{k}$ are edge-disjoint subgraphs of $K_{n}$. Thus there is a $k$-decomposition $\left(G_{1}, \ldots, G_{k}\right)$ of $K_{n}$ such that $H_{i} \subseteq G_{i}$ for each $i$. We have $\sigma\left(G_{i}\right) \geq \delta\left(H_{i}\right)+1 \geq p m+1$. Hence,

$$
\sigma\left(k ; K_{n}\right) \geq k(p m+1)=p n+k \geq(\sqrt{k}-1) n+k .
$$

The construction in the proof of Theorem 5 works only for special values of $k$. For small values of $k$, there are other natural candidates for $\sigma$-optimal $k$-decompositions of $K_{n}$.

Construction: Consider $n=m(k-1)+1$, with $k \geq 2$ and $m \geq 1$. Let $V_{1} \cup \ldots \cup V_{k-1} \cup\{v\}$ be a partition of an $n$-set into $m$-sets and a singleton. For $1 \leq i \leq k-1$, let $G_{i}$ be the complete graph with vertex set $V_{i} \cup\{v\}$. Let $G_{k}$ be the complete $(k-1)$-partite graph with color classes $V_{1}, \ldots, V_{k-1}$. The $k$-decomposition $\left(G_{1}, \ldots, G_{k}\right)$ of $K_{n}$ yields $\sigma\left(k ; K_{n}\right) \geq(k-1) m+(k-2) m+k$.

For general $n$, let $m=\left\lfloor\frac{n-1}{k-1}\right\rfloor$ and $r=n-1-m(k-1)$. Form $G_{1}, \ldots, G_{k}$ as above, except enlarge each of $V_{1}, \ldots, V_{r}$ by one vertex. Since $m=\frac{n-1-r}{k-1}$, this 
yields $\sigma\left(k ; K_{n}\right) \geq(2 k-3) \frac{n-1-r}{k-1}+2 r-2+k=(2 k-3) \frac{n-1}{k-1}-\frac{k-2}{k-1}+k$. Since $\sigma\left(k ; K_{n}\right)$ is an integer, we obtain

$$
\sigma\left(k ; K_{n}\right) \geq\left\lfloor\frac{2 k-3}{k-1}(n-1)\right\rfloor+k .
$$

In particular, for $n \geq 1$ we have $\sigma\left(2 ; K_{n}\right) \geq n+1, \sigma\left(3 ; K_{n}\right) \geq\lfloor(3 n+3) / 2\rfloor$, and $\sigma\left(4 ; K_{n}\right) \geq\lfloor(5 n+7) / 3\rfloor$.

The following upper bound on $\sigma\left(k ; K_{n}\right)$ shows that this construction is optimal for $k \leq 4$.

Theorem 10 If $k \geq 2$ and $n \geq 2$, then $\sigma\left(k ; K_{n}\right) \leq f(k, n)$, where

$$
f(k, n)= \begin{cases}n+1, & \text { if } k=2 \\ (3 n+3) / 2, & \text { if } k=3 \\ (5 n+7) / 3, & \text { if } k=4 \\ (k-1)(n+1) / 2, & \text { if } k \geq 5\end{cases}
$$

Proof: The proof is by induction on $n$. For $n=2$ and $k \geq 2$, we have $\sigma\left(k ; K_{2}\right)=k+1 \leq f(k, 2)$.

For $n \geq 3$, consider a $\sigma$-optimal $k$-decomposition $\left(G_{1}, \ldots, G_{k}\right)$ of $K_{n}$. Let $H_{i}$ be a $\sigma$-critical subgraph of $G_{i}$ with $\sigma\left(H_{i}\right)=\sigma\left(G_{i}\right)$, so $\sigma\left(G_{i}\right)=\delta\left(H_{i}\right)+1$. Let $n_{i}=\left|V\left(H_{i}\right)\right|$ and $\delta_{i}=\delta\left(H_{i}\right)$.

First we prove for distinct $i$ and $j$ in $[k]$ that

$$
\delta_{i}+\delta_{j} \leq n-1
$$

If $H_{i}$ and $H_{j}$ are disjoint, then $\delta_{i}+\delta_{j} \leq\left(n_{i}-1\right)+\left(n_{j}-1\right) \leq n-2$. If they have a common vertex $x$, then $\delta_{i}+\delta_{j} \leq d_{H_{i}}(x)+d_{H_{j}}(x) \leq n-1$. This proves (3) and the case $k=2$. Now consider $k \geq 3$.

Case 1 Some vertex $x$ of $K_{n}$ lies in at most one of $H_{1}, \ldots, H_{k}$. Here criticality of $H_{i}$ and the induction hypothesis yield

$$
\begin{aligned}
\sigma\left(k ; K_{n}\right) & =\sum_{i=1}^{k} \delta\left(H_{i}\right)+k \leq \sum_{i=1}^{k} \delta\left(H_{i}-x\right)+k+1 \\
& \leq \sigma\left(k ; K_{n-1}\right)+1 \leq f(k, n-1)+1<f(k, n) .
\end{aligned}
$$

Case 2 Every vertex of $K_{n}$ belongs to exactly two of $H_{1}, \ldots, H_{k}$. Consider distinct $i$ and $j$ in $[k]$. Let $X_{i j}=V\left(H_{i}\right) \cap V\left(H_{j}\right)$ and $n_{i j}=n_{j i}=\left|X_{i j}\right|$. Furthermore, let $\delta_{i j}=\delta\left(H_{i}\left[X_{i j}\right]\right)$ if $n_{i j} \neq 0$ and $\delta_{i j}=-1$ otherwise. Since $H_{i}\left[X_{i j}\right]$ and $H_{j}\left[X_{i j}\right]$ decompose the subgraph induced by $X_{i j}$, the case $k=2$ yields $\delta_{i j}+\delta_{j i} \leq n_{i j}-1$. 
For $i \neq j$ and $S_{i j}=[k]-\{i, j\}$, a vertex $x$ of minimum degree in $H_{i}\left[X_{i j}\right]$ yields $\delta_{i} \leq d_{H_{i}}(x) \leq \delta_{i j}+\sum_{\ell \in S_{i j}} n_{i \ell}$. Summing over all ordered pairs $(i, j)$ yields

$$
(k-1) \sum_{i=1}^{k} \delta_{i} \leq \sum_{1 \leq i<j \leq k}\left(n_{i j}-1\right)+2(k-2) \sum_{1 \leq i<j \leq k} n_{i j}=(2 k-3) n-\left(\begin{array}{l}
k \\
2
\end{array}\right)
$$

and hence

$$
\sigma\left(k ; K_{n}\right)=k+\sum_{i=1}^{k} \delta_{i} \leq k+\frac{2 k-3}{k-1} n-\frac{k}{2} \leq \frac{2 k-3}{k-1} n+\frac{k}{2}
$$

It is easily checked that $\left\lfloor\frac{2 k-3}{k-1} n+\frac{k}{2}\right\rfloor \leq f(k, n)$ for $k \geq 3$ and $n \geq 2$.

Case 3 Some vertex $x$ of $K_{n}$ belongs to at least three of $H_{1}, \ldots, H_{k}$. Indexing the decomposition so that $\left\{i: x \in V\left(H_{i}\right)\right\}=[m]$, we have $\delta_{1}+\delta_{2}+\ldots+\delta_{m} \leq n-1$. If $m=k$, we are done, so we may assume that $k \geq 4$. If $m \leq k-2$, then applying (3) to $G-x$ states for $i, j \in[k]-[m]$ that

$$
\delta_{i}+\delta_{j} \leq n-2 .
$$

Summing over all such pairs yields $\sum_{i=m+1}^{k} \delta_{i} \leq(k-m)(n-2) / 2$. Since $m \geq 3$ and $k \geq 4$, this implies that

$$
\begin{aligned}
\sigma\left(k ; K_{n}\right) & \leq k+(n-1)+\frac{(k-m)(n-2)}{2} \\
& \leq k+n-1+\frac{(k-3)(n-2)}{2}=\frac{1}{2}(k-1) n+2 \leq f(k, n) .
\end{aligned}
$$

Finally, if $m=k-1$ and $\delta_{1} \leq \ldots \leq \delta_{m}$, then $\delta_{1}+\ldots+\delta_{m-1} \leq \frac{m-1}{m}(n-1)$. By (3), $\delta_{m}+\delta_{k} \leq n-1$. Hence,

$$
\sigma\left(k ; K_{n}\right) \leq k+\frac{m-1}{m}(n-1)+n-1=k+\frac{2 k-3}{k-1}(n-1) .
$$

It is easily checked that $\left\lfloor\frac{2 k-3}{k-1}(n-1)+k\right\rfloor \leq f(k, n)$ for $k \geq 4$ and $n \geq 2$.

The Construction and Theorem 10 together complete the computation of $\sigma\left(k ; K_{n}\right)$ for $k \leq 4$

Corollary 11 If $n$ is a positive integer, then $\sigma\left(2 ; K_{n}\right)=n+1, \sigma\left(3 ; K_{n}\right)=$ $\left\lfloor\frac{3}{2} n+\frac{3}{2}\right\rfloor$ and $\sigma\left(4 ; K_{n}\right)=\left\lfloor\frac{5}{3} n+\frac{7}{3}\right\rfloor$. 


\section{Chromatic number of $r$-uniform hypergraphs}

We next obtain an upper bound for $\chi\left(k ; K_{n}^{r}\right)$. Again we need an auxiliary result.

Proposition 12 If $G$ is an $r$-uniform hypergraph with $n$ vertices, then

$$
\chi(G) \leq 1+\frac{\omega(G)}{r-1}+\frac{n-\omega(G)}{r} .
$$

Proof: We produce a proper coloring. If $V(G)$ is not a clique, then it has an independent set of size $r$; choose color classes of size $r$ until the vertex set $X$ that remains is a clique. Let $s=(n-|X|) / r$.

Since $X$ is a clique, we have $|X| \leq \omega(G)$. Since sets of size $r-1$ contain no edge, $G[X]$ is $q+1$-colorable, where $q=\left\lfloor\frac{|X|}{r-1}\right\rfloor$. Thus $\chi(G) \leq s+q+1$.

Since $\frac{q}{r} \leq \frac{\omega(G)}{r(r-1)}=\omega(G)\left(\frac{1}{r-1}-\frac{1}{r}\right)$ and $r s+(r-1) q \leq n$, we have

$$
s+q \leq \frac{n}{r}-\frac{r-1}{r} q+q \leq \frac{n+q}{r} \leq \frac{n-\omega(G)}{r}+\frac{\omega(G)}{r-1} .
$$

Now we are ready to prove Theorem 6 .

Theorem 6 If $k$ and $r$ are positive integers with $r \geq 2$, then there exists an integer $c_{k, r}$ such that, for every positive integer $n$,

$$
\chi\left(k ; K_{n}^{r}\right) \leq \frac{n}{r-1}+c_{k, r}
$$

Proof: Let $\left(G_{1}, \ldots, G_{k}\right)$ be an arbitrary $k$-decomposition of the $r$-uniform complete hypergraph $K_{n}^{r}$. As in Theorem 3, we define a sequence of vertex subsets. Let $X_{0}=\emptyset$. For $j \geq 1$, let $X_{j}$ be a maximum clique among the induced subhypergraphs $G_{1}-\bigcup_{i=0}^{j-1} X_{i}, \ldots, G_{k}-\bigcup_{i=0}^{j-1} X_{i}$.

If $\left|X_{1}\right|<k r(r-1)$, then $n<R$, where $R$ is the Ramsey number $R_{k}^{r}(k r(r-$ $1), \ldots, k r(r-1))$. Since $\sum_{i=1}^{k} \chi\left(G_{i}\right) \leq k n$, we have $\sum_{i=1}^{k} \chi\left(G_{i}\right)<k R$, and it suffices to have $c_{k, r} \geq k R$ in this case.

Otherwise, there is a largest positive integer $s$ such that $\left|X_{j}\right| \geq k r(r-1)$ for $1 \leq j \leq s$. Let $X=\bigcup_{j=1}^{s} X_{j}$. For $1 \leq i \leq k$, let $Y_{i}=\bigcup\left\{X_{j}: X_{j}\right.$ is a clique in $G_{i}$ and $\left.j \leq s\right\}$. Let $n_{i}=\left|Y_{i}\right|, G_{i}^{\prime}=G_{i}\left[Y_{i}\right], \omega_{i}=\omega\left(G_{i}^{\prime}\right)$, and $\omega^{*}=\sum_{i=1}^{k} \omega_{i}$. The sets $Y_{1}, \ldots, Y_{k}$ are pairwise disjoint and have union $X$. Furthermore, $\omega_{i}=\left|X_{j}\right|$ for some $X_{j} \subseteq Y_{i}$. Since $\left|X_{j}\right| \geq k r(r-1)$ for $j \leq s$, we conclude that $|X|-\omega^{*} \geq$ $(s-k) k r(r-1)$.

By Proposition 12, $\chi\left(G_{i}^{\prime}\right) \leq \frac{\omega_{i}}{r-1}+\frac{n_{i}-\omega_{i}}{r}+1$. We rewrite the upper bound as $\frac{n_{i}}{r-1}-\frac{n_{i}-\omega_{i}}{r(r-1)}+1$. Summing the upper bounds yields

$$
\sum_{i=1}^{k} \chi\left(G_{i}^{\prime}\right) \leq \frac{|X|}{r-1}-\frac{|X|-\omega^{*}}{r(r-1)}+k \leq \frac{n}{r-1}-k(s-k)+k .
$$


A clique in $G_{i}$ is an independent set in $G_{j}$ for $j \neq i$. Therefore, the inequality above implies

$$
\sum_{i=1}^{k} \chi\left(G_{i}[X]\right) \leq \sum_{i=1}^{k} \chi\left(G_{i}^{\prime}\right)+(k-1) s \leq \frac{n}{r-1}+k^{2}+k .
$$

By construction, $\omega\left(G_{i}-X\right)<k r(r-1)$. Therefore, $\chi\left(G_{i}-X\right) \leq n-|X|<R$. By the subadditivity of $\chi$, this yields

$$
\sum_{i=1}^{k} \chi\left(G_{i}\right) \leq \frac{n}{r-1}+k^{2}+k+k R
$$

which proves the claim with $c_{k, r}=k^{2}+k+k R$.

\section{Graphs on surfaces}

This section considers graphs embedded on a surface $\Sigma$. For a graph parameter $p$ and a positive integer $k$, let $p(k ; \Sigma)=\max \{p(k ; G): G$ embeds on $\Sigma\}$.

Surfaces can be classified by their genus and orientability. For $h \geq 0$, the orientable surface $\Sigma_{h}$ is obtained by adding $h$ handles to a sphere. For $h \geq 1$, the non-orientable surface $\Pi_{h}$ is obtained from a sphere with $h$ holes by attaching $h$ Möbius bands along their boundaries to the boundaries of the holes. For example, $\Pi_{1}$ is the projective plane, $\Pi_{2}$ is the Klein bottle, etc. The Euler genus $g(\Sigma)$ of the surface $\Sigma$ is $2 h$ if $\Sigma=\Sigma_{h}$ and is $h$ if $\Sigma=\Pi_{h}$. The Euler characteristic of $\Sigma$ is $2-g(\Sigma)$.

For a simple graph $G$ with vertex set $V$ and edge set $E$ embedded on a surface $\Sigma$ of Euler genus $g$, Euler's Formula states that $|V|-|E|+|F| \geq 2-g$, where $F$ is the set of faces, with equality holding if and only if every face is a 2-cell. When $|V| \geq 3$, this yields $|E| \leq 3|V|-6+3 g$. For $g \geq 1$, this implies that every subgraph of $G$ has a vertex of degree at most $H(g)-1$, where

$$
H(g)=\left\lfloor\frac{7+\sqrt{24 g+1}}{2}\right\rfloor .
$$

In particular, $\sigma(G) \leq H(g)$. Consequently, if $g \geq 1$, then

$$
\omega(G) \leq \chi(G) \leq \chi_{\ell}(G) \leq \sigma(G) \leq H(g) .
$$

For every surface $\Sigma$ other than the Klein bottle, the Heawood number $H(g)$ is, in fact, the maximum chromatic number of graphs embeddable on $\Sigma$, attained by $K_{H(g)}$. This landmark result conjectured by Heawood [11] was proved by Ringel [18] and Ringel-Youngs [17]. Furthermore, every graph with chromatic number 
$H(g)$ embedded on $\Sigma$ contains a complete graph on $H(g)$ vertices as a subgraph. This was proved by Dirac $[4,5]$ for the torus and for $g \geq 4$ and was proved by Albertson and Hutchinson [1] for $g \in\{1,3\}$.

Although $H(2)=7$, Franklin [10] proved that the maximum chromatic number for the Klein bottle is 6 . Furthermore, there are 6-chromatic graphs on the Klein bottle not containing $K_{6}$. Such a graph appears in [1].

The version of Brooks' Theorem for list-chromatic number implies that if $G$ is a graph on the Klein bottle, then also $\chi_{\ell}(G) \leq 6$. For graphs on the sphere, the maximum chromatic number is 4 , but the maximum list-chromatic number is 5 (upper bound by Thomassen [21], lower bound by Voigt [22]).

Further results about the chromatic number of graphs embedded on given surfaces appear in the book of Jensen and Toft [13].

For a surface $\Sigma$ and a positive integer $k$, we have the familiar inequalities

$$
\omega(k ; \Sigma) \leq \chi(k ; \Sigma) \leq \chi_{\ell}(k ; \Sigma) \leq \sigma(k ; \Sigma) .
$$

When a graph embeds on the sphere $\Sigma_{0}$, the disjoint union of $k$ copies of $G$ also embeds on $\Sigma_{0}$. Hence $\sigma\left(k ; \Sigma_{0}\right)=6 k$ and $\omega\left(k ; \Sigma_{0}\right)=\chi\left(k ; \Sigma_{0}\right)=4 k$. For all other surfaces, this is not true. We begin by establishing a lower bound for $\omega(k ; \Sigma)$.

Theorem 13 Let $\Sigma$ be a surface with positive Euler genus $g$.

(a) If $\Sigma$ is orientable, then $\omega(k ; \Sigma) \geq k H(2\lfloor g / 2 k\rfloor)$.

(b) If $\Sigma$ is non-orientable and $\lfloor g / k\rfloor \geq 3$, then $\omega(k ; \Sigma) \geq k H(\lfloor g / k\rfloor)$.

Proof: If $\Sigma$ is orientable, then let $g^{\prime}=2\lfloor g / 2 k\rfloor$ and $m=H\left(g^{\prime}\right)$; since $g^{\prime}$ is even, $K_{m}$ embeds on an orientable surface with Euler genus $g^{\prime}$. If $\Sigma$ is non-orientable, then let $g^{\prime}=\lfloor g / k\rfloor$ and $m=H\left(g^{\prime}\right)$; since $g^{\prime} \geq 3, K_{m}$ embeds on a non-orientable surface with Euler genus $g^{\prime}$.

In either case, let $G$ be the disjoint union of $k$ copies of $K_{m}$. Since $k g^{\prime} \leq g$, it then follows (see [14]) that $G$ embeds on $\Sigma$. Thus $\omega(k ; G) \geq k m$.

For a surface $\Sigma$ of Euler genus $g$, this lower bound on $\omega(k ; \Sigma)$ is approximately $\left(7 k+\sqrt{24 g k+k^{2}}\right) / 2$. We next establish an upper bound on $\sigma(k ; \Sigma)$ that is asymptotic to this for fixed $k$ and large $g$.

Theorem 14 If $\Sigma$ is a surface with positive Euler genus $g$, then

$$
\sigma(k ; \Sigma) \leq\left\lfloor\frac{7 k+\sqrt{24 k g+49 k^{2}-48 k}}{2}\right\rfloor .
$$


Proof: Given a graph $G$ embedded on $\Sigma$, let $\left(G_{1}, \ldots, G_{k}\right)$ be a $k$-decomposition of $G$. For each $i$, let $H_{i}$ be a $\sigma$-critical subgraph of $G_{i}$ with $\sigma\left(H_{i}\right)=\sigma\left(G_{i}\right)$, and let $d_{i}=\delta\left(H_{i}\right)$, so $\sigma\left(G_{i}\right)=d_{i}+1$.

We may assume that $d_{1} \geq \ldots \geq d_{k}$. Let $s$ be the unique nonnegative integer such that $d_{i}>5$ if and only if $i \leq s$. If $s=0$, then

$$
\sum_{i=1}^{k} \sigma\left(G_{i}\right) \leq 5 k \leq\left\lfloor\frac{7 k+\sqrt{24 k g+49 k^{2}-48 k}}{2}\right\rfloor .
$$

If $s \geq 1$, then let $H=\bigcup_{j \in[s]} H_{j}$. Let $n=|V(H)|$ and $e=|E(H)|$. For $I \subseteq[s]$, denote by $V_{I}$ the set of all vertices of $H$ that belong to each graph $H_{i}$ with $i \in I$ and to no graph $H_{i}$ with $i \in[s]-I$. Let $n_{I}=\left|V_{I}\right|$ and $n_{i}=n_{\{i\}}$. Thus,

$$
\left|V\left(H_{i}\right)\right|=\sum_{i \in I \subseteq[s]} n_{I} \quad \text { and } \quad n=\sum_{I \subseteq[s]} n_{I} .
$$

Since $s \geq 1$, we have $n \geq 7$. Since $H \subseteq G$, also $H$ embeds on $\Sigma$. By Euler's Formula, $6 n+6 g-12 \geq 2 e$. Every vertex of $V_{I}$ has degree at least $\sum_{i \in I} d_{i}$. Thus,

$$
6 \sum_{I \subseteq[s]} n_{I}+6 g-12 \geq 2 e \geq \sum_{I \subseteq[s]} n_{I} \sum_{i \in I} d_{i} .
$$

By rearranging the inequality and interchanging the order of summation (subtracting more copies of 6 when $|I|>1$ ), we obtain

$6 g-12 \geq \sum_{I \subseteq[s]} n_{I}\left(-6+\sum_{i \in I} d_{i}\right) \geq \sum_{i=1}^{s}\left(\left(d_{i}-6\right) \sum_{i \in I \subseteq[s]} n_{I}\right)=\sum_{i=1}^{s}\left(d_{i}-6\right)\left|V\left(H_{i}\right)\right|$.

Since $\left|V\left(H_{i}\right)\right| \geq d_{i}+1$ and $d_{i} \geq 6$ for $i \in[s]$, we have

$$
6 g-12 \geq \sum_{i=1}^{s}\left(d_{i}-6\right)\left(d_{i}+1\right)=\sum_{i=1}^{s}\left(\left(d_{i}-\frac{5}{2}\right)^{2}-\frac{49}{4}\right) .
$$

Consequently,

$$
6 g-12+\frac{49}{4} s \geq \sum_{i=1}^{s}\left(d_{i}-\frac{5}{2}\right)^{2} \geq \frac{1}{s}\left(\sum_{i=1}^{s}\left(d_{i}-\frac{5}{2}\right)\right)^{2}=\frac{1}{s}\left(\sum_{i=1}^{s} d_{i}-s \frac{5}{2}\right)^{2},
$$

and therefore

$$
\sum_{i=1}^{s} d_{i} \leq \frac{1}{2}\left(5 s+\sqrt{24 s g+49 s^{2}-48 s}\right) .
$$

For $i>s$, we have $d_{i} \leq 5$. Thus we conclude that

$\sum_{i=1}^{k} \sigma\left(G_{i}\right)=k+\sum_{i=1}^{k} d_{i} \leq 6 k-5 s+\sum_{i=1}^{s} d_{i} \leq \frac{1}{2}\left(12 k-5 s+\sqrt{24 s g+49 s^{2}-48 s}\right)$. 
This upper bound increases with $s$ in the domain $s \geq 0$. Since $s \leq k$, we thus set $s=k$ to obtain

$$
\sum_{i=1}^{k} \sigma\left(G_{i}\right) \leq\left\lfloor\frac{7 k+\sqrt{24 k g+49 k^{2}-48 k}}{2}\right\rfloor .
$$

This completes the proof.

\section{References}

[1] M. O. Albertson and J. P. Hutchinson, The three excluded cases of Dirac's map-color theorem, Ann. New York Acad. Sci. 319 (1979), 7-17.

[2] N. Alon, Choice numbers of graphs: a probabilistic approach, Combinatorics, Probability and Computing 1 (1992), 1007-114.

[3] J. Bosák, Decompositions of Graphs, Kluwer Academic Publishers (Mathematics and Its Application, Volume 47), Dordrecht, 1990.

[4] G. A. Dirac, Map colour theorems related to the Heawood colour formula, J. London Math. Soc. 31 (1956), 460-471.

[5] G. A. Dirac, Short proof of a map-colour theorem, Canad. J. Math. 9 (1957), 225-226.

[6] R. Diestel, Graph Theory, Springer-Verlag, New York, 1997.

[7] P. Erdös and A. Hajnal, On chromatic number of graphs and set setsystems. Acta Math. Acad. Sci. Hungar. 17 (1966), 61-99.

[8] P. Erdös, A.L. Rubin and H. Taylor, Choosability in graphs, Proc. West Coast Conf. on Combinatorics, Graph Theory and Computing, Congressus Numerantium XXVI (1979), 125-157.

[9] H. J. Finck, Über die chromatische Zahl eines Graphen und seines Komplementes (I), Wiss. Z. der TU Ilmenau 12 (1966), 243-246.

[10] P. Franklin, A six-color problem, J. Math. Phys. 13 (1934), 363-369.

[11] P. J. Heawood, Map colour theorem, Quart. J. Pure Appl. Math. 24 (1890), 332-338.

[12] S. Janson, T. Łuczak and A. Rucinski, Random Graphs, John Wiley \& Sons, New York, 2000. 
[13] T. R. Jensen and B. Toft, Graph Coloring Problems Wiley-Interscience Series in Discrete Mathematics and Optimization, John Wiley \& Sons, New York, 1995.

[14] B. Mohar and C. Thomassen, Graphs on Surfaces, Johns Hopkins University Press, Baltimore, 2001.

[15] E. A. Nordhaus and J. W. Gaddum, On complementary graphs, Amer. Math. Monthly 63 (1956), 175-177.

[16] J. Plesník, Bounds on the chromatic numbers of multiple factors of a complete graph, J. Graph Theory 2 (1978), 9-17.

[17] G. Ringel and J. W. T. Youngs, Solution of the Heawood map-coloring problem, Proc. Nat. Acad. Sci. U.S.A. 60 (1968), 438-445.

[18] G. Ringel, Map Color Theorem, Springer-Verlag, New York, 1974.

[19] F. P. Ramsey, On a problem of formal logic, Proc. London Math. Soc. 30 (1930), 264-286.

[20] G. Szekeres and H. S. Wilf, An inequality for the chromatic number of a graph, J. Combin. Theory Ser. B 4 (1968), 1-3.

[21] C. Thomassen, Every planar graph is 5-choosable, J. Combin. Theory Ser. B 62 (1994), 180-181.

[22] M. Voigt, List colorings of planar graphs, Discrete Math. 120 (1993), 215219.

[23] T. Watkinson, A theorem of the Nordhaus-Gaddum class, Ars Combinatoria 20-B (1985), 35-42. 